\title{
Efficacy of Nifekalant Hydrochloride in the Treatment of Fatal Ventricular Arrhythmia in Patients With Ischemic Heart Disease
}

\author{
Jiro Ando, ${ }^{1} \mathrm{MD}$, Mikio KAKISHITA, ${ }^{1} \mathrm{MD}$, Koyu SAKAI, ${ }^{1} \mathrm{MD}$, \\ Yasuo KomurA, ${ }^{1} \mathrm{MD}$, Kei NishIYAMA, ${ }^{1} \mathrm{MD}$, Masashi IwABUCHI, ${ }^{1} \mathrm{MD}$, \\ Hiroyoshi Yokor, ${ }^{1} \mathrm{MD}$, Hitoshi Yasumoto,${ }^{1} \mathrm{MD}$, Hideyuki NosAKA, ${ }^{1} \mathrm{MD}$, \\ and Masakiyo NoBUYOSHI, ${ }^{1} \mathrm{MD}$
}

\section{SUMMARY}

Ventricular tachycardia (VT), which causes hemodynamic instability, and ventricular fibrillation (VF) sometimes occur in patients with severe underlying cardiovascular disease such as myocardial ischemia or infarction, and are associated with high mortality. This report presents the efficacy of nifekalant hydrochloride (nifekalant), a pure class III antiarrhythmic agent, in treating life-threatening ventricular arrhythmia in such patients.

From June 2000, when nifekalant became commercially available in Japan, to May 2003, 30 ischemic heart disease (IHD) patients with VT/VF resistant to direct-current (DC) countershock received nifekalant in our hospital. These 30 patients served as the nifekalant group in this study. As a control group, we also included 33 IHD patients with VT/VF that had been resistant to DC countershock upon or during hospitalization between January 1996 and May 2000 before nifekalant became commercially available.

No significant differences were observed in patient background factors and treatments between the two groups. The rates of death within 48 hours of occurrence of VT/VF were significantly lower in the nifekalant group $(7 \%, 2 / 30)$ than in the control group $(27 \%, 9 /$ 33; $P<0.03)$. The rates of cardiac death during hospitalization were also significantly lower in the nifekalant group $(40 \%, 12 / 30)$ than in the control group $(67 \%, 22 / 33 ; P<$ 0.03 ). The rates of survival until hospital discharge were significantly higher in the nifekalant group $(57 \%, 17 / 30)$ than in the control group $(30 \%, 10 / 33 ; P<0.03)$. Multivariate analysis of all 63 patients revealed nifekalant administration was the factor that significantly improved the mortality (odds ratio for cardiac death, 0.26 ; $95 \%$ confidence interval $(\mathrm{CI}), 0.07$ to $0.95 ; P=0.041)$.

Nifekalant improves the prognosis for life-threatening ventricular arrhythmia in IHD patients. (Int Heart J 2005; 46: 647-656)

Key words: Nifekalant, Class III antiarrhythmic agents, Ventricular arrhythmia, Ischemic heart disease

From the ${ }^{1}$ Department of Cardiology, Kokura Memorial Hospital, Fukuoka, Japan.

Address for correspondence: Jiro Ando, MD, Department of Cardiology, Kokura Memorial Hospital, 1-1 Kifunemachi, Kokurakita-ku, Kitakyushu, Fukuoka 802-8555, Japan.

Received for publication August 6, 2004.

Revised and accepted March 11, 2005. 
THE arrhythmias that occur in ischemic heart disease are diverse. Some are refractory, especially in patients with deterioration of cardiac function, heart failure, and unstable hemodynamics. As some reports have shown that defibrillation during the early phase after ventricular fibrillation (VF) increased the rate of survival to hospital discharge ${ }^{1-5)}$ it is considered that treatment of severe ventricular arrhythmia has important effects on the prognosis of patients with ischemic heart disease (IHD).

Nifekalant hydrochloride (nifekalant), a pure potassium channel blocker, ${ }^{6,7)}$ was developed in Japan and approved in 1999 for clinical use to treat life-threatening, refractory fatal ventricular arrhythmias. Nifekalant, a new chemical compound with a pyrimidinedione backbone, is an antiarrhythmic drug categorized as a class III agent according to the Vaughan Williams classification. ${ }^{6,8,9)}$

Nifekalant mainly inhibits Ikr, a delayed rectifier potassium channel. ${ }^{6,10)}$ By inhibiting Ikr and regulating action potential duration, ${ }^{6}$ nifekalant prolongs the refractory period of ventricular and atrial muscles, ${ }^{11-14)}$ thereby reducing the induction of reentry arrhythmia ${ }^{8,11)}$ and lowering the defibrillation threshold. ${ }^{15)}$ We evaluated the efficacy of nifekalant in treating fatal ventricular arrhythmia.

\section{METHODS}

Study population: We examined 30 patients who were administered nifekalant for resistance to direct-current (DC) countershock out of 127 IHD patients with ventricular tachycardia (VT)/VF upon or during hospitalization between June 2000 and May 2003. The control group was comprised of 33 IHD patients with VT/VF resistant to DC countershock upon or during hospitalization between January 1996 and May 2000, when nifekalant had not yet been approved.

Definitions and in-hospital outcome: The medical records of each group were reviewed. We defined resistance to DC countershock for VT/VF as being when VT/VF could not be terminated by first DC countershock. Coronary angiography was performed in 59 out of $63(94 \%)$ patients to diagnose IHD before or after the onset of VT/VF. In this study, acute myocardial infarction (AMI) and unstable angina pectoris were dealt with as acute coronary syndrome (ACS). Myocardial infarction and angina pectoris were diagnosed based on the symptoms, 12-lead electrocardiogram, coronary angiography, and the elevation of cardiac enzymes. Patients were considered to have an old myocardial infarction (OMI) if previously diagnosed by a 12-lead electrocardiogram or coronary angiography. Heart failure was diagnosed by chest $\mathrm{X}$-ray or hemodynamic measurement. We defined a low ejection fraction (EF) as being $\mathrm{EF} \leq 35 \%$ by ultrasound cardiogram.

The validity of nifekalant was defined as follows: we defined the termination effect when VT/VF terminated within 5 minutes after starting nifekalant 
administration without DC countershock, and the preventive effect when VT/VF was interrupted by DC countershock during nifekalant administration. We also examined the number of DC countershocks delivered as an index of the efficacy of nifekalant.

Mortality within 48 hours of occurrence of VT/VF, percentage cardiac death, and survival to hospital discharge were compared in the nifekalant and control groups. Death-related factors were also analyzed for all 63 cases.

Statistical analysis: All statistical comparisons were performed with StatView Version 5.0 software (SAS Institute Inc. Cary, NC, USA.). Continuous variables are displayed as the mean \pm standard deviation (SD), and were compared with a $t$-test. Categorical data are presented as frequencies and were compared with the chi-square test. All statistical tests were two-tailed, with values of $P<0.05$ considered significant. The multivariate analysis included factors identified by univariate analysis as associated with death-related factors.

\section{RESUlts}

Patient background: The patient backgrounds and therapies the subjects received in the nifekalant and control groups are shown in Tables I and II. No significant differences in patient background factors were observed between the nifekalant and control groups (Table I), nor were there any significant differences observed between the groups with respect to the therapies the subjects received (Table II).

Table I. Clinical Characteristics of Patients

\begin{tabular}{lccc}
\hline & $\begin{array}{c}\text { Nifekalant group } \\
n=30\end{array}$ & $\begin{array}{c}\text { Control group } \\
n=33\end{array}$ & $P$ value \\
\hline Age $>75$ y.o & $5(17 \%)$ & $11(33 \%)$ & NS \\
Male & $21(70 \%)$ & $22(67 \%)$ & NS \\
HTN & $14(47 \%)$ & $17(52 \%)$ & NS \\
HLP & $8(27 \%)$ & $5(15 \%)$ & NS \\
DM & $10(33 \%)$ & $13(39 \%)$ & NS \\
Cre $>2.0 \mathrm{mg} / \mathrm{dL}$ & $9(30 \%)$ & $8(24 \%)$ & NS \\
HF & $23(77 \%)$ & $28(85 \%)$ & NS \\
LVEF $\leqq 35 \%$ & $12(40 \%)$ & $14(42 \%)$ & NS \\
ACS & $22(73 \%)$ & $24(73 \%)$ & NS \\
OMI & $15(50 \%)$ & $15(45 \%)$ & NS \\
LMTD & $6(20 \%)$ & $4(12 \%)$ & NS \\
MVD & $15(50 \%)$ & $17(52 \%)$ & \\
\hline
\end{tabular}

HTN = hypertension; HLP = hyperlipidemia; DM = diabetes mellitus; Cre = creatinine; $\mathrm{HF}=$ heart failure; $\mathrm{LVEF}=$ left ventricular ejection fraction; $\mathrm{ACS}=$ acute coronary syndrome; $\mathrm{OMI}=$ old myocardial infarction; $\mathrm{LMTD}=$ left main trunk disease; $\mathrm{MVD}=$ multivessel coronary disease. 
Table II. Treatments of Patients

\begin{tabular}{lccc}
\hline & $\begin{array}{c}\text { Nifekalant group } \\
n=30\end{array}$ & $\begin{array}{c}\text { Control group } \\
n=33\end{array}$ & $P$ value \\
\hline Administration of lidocaine & $26(87 \%)$ & $30(91 \%)$ & NS \\
${\text { Administration of } \mathrm{MgSO}_{4}}_{\text {Pressor treatment* }}^{15(50 \%)}$ & $9(27 \%)$ & NS \\
Revascularization therapy** & $23(77 \%)$ & $31(94 \%)$ & NS \\
Assisted circulation*** & $24(80 \%)$ & $29(88 \%)$ & NS \\
Pacing therapy & $16(53 \%)$ & $20(61 \%)$ & NS \\
& $12(40 \%)$ & $8(24 \%)$ & NS \\
\hline
\end{tabular}

$\mathrm{MgSO}_{4}=$ magnesium sulfate.

*Pressor treatment indicates administration of pressor agents.

**Revascularization therapy indicates performance of percutaneous coronary intervention or coronary artery bypass grafting.

***Assisted circulation indicates use of intra-aortic balloon pumping and/or percutaneous cardiopulmonary support.

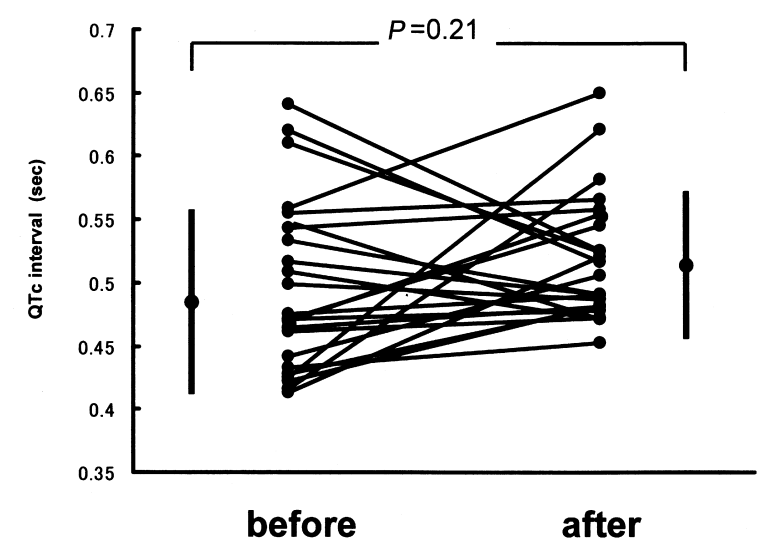

Figure 1. Changes in QTc interval. QTc interval was measured before and after administration of nifekalant in each patient. QTc was calculated by Bazett's formula: $\mathrm{QTc}=\mathrm{QT} / \mathrm{RR} 1 / 2$. Values are mean $\pm \mathrm{SD}, n=24$, paired $t$ test.

Drug administration: Nifekalant was administered intravenously to all subjects in the nifekalant group. The mean loading and maintenance doses of nifekalant infusion were $0.27 \mathrm{mg} / \mathrm{kg} \mathrm{BW}$ and $0.26 \mathrm{mg} / \mathrm{kg} \mathrm{BW} / \mathrm{h}$, respectively. The mean duration of administration was 5.6 days, with a maximum of 48 days.

Corrected QT intervals: We measured corrected QT (QTc) intervals before and after administration of nifekalant in 24 patients in the nifekalant group (Figure 1). We could not measure QTc intervals in 6 out of 30 patients because their initial electrocardiogram records were VT or VF. No significant differences were 


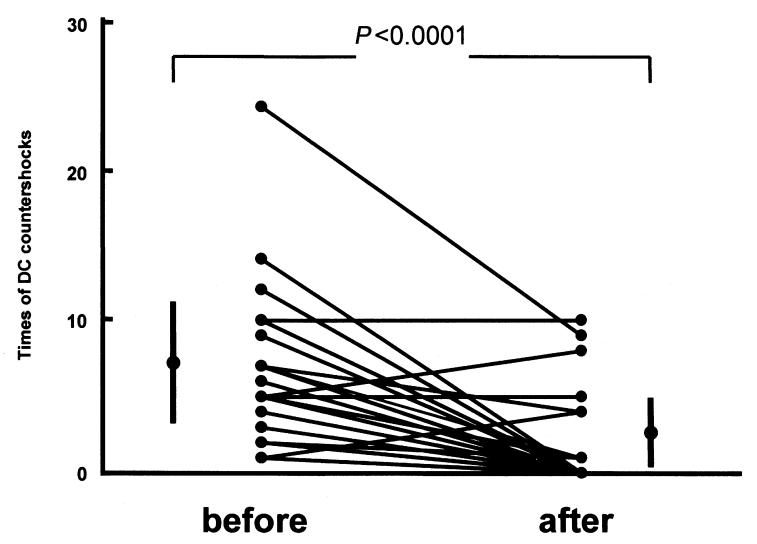

Figure 2. Times of DC countershocks received before and after administration of nifekalant in each patient. DC $=$ direct-current. Values are mean \pm $\mathrm{SD}, n=30$, paired $t$-test.

observed between before and after administration of nifekalant with respect to the change in QTc interval (before versus after $=0.49$ seconds \pm 0.14 versus 0.52 seconds $\pm 0.10, P=0.21)$.

Number of DC countershocks delivered: Figure 2 shows the number of DC countershocks delivered before and after nifekalant administration in all 30 cases in the nifekalant group. A significant decrease in the number of DC countershocks was observed after administration of nifekalant (before versus after $=6.8 \pm 4.9$ versus $1.5 \pm 2.9, P<0.0001)$.

Efficacy rate: Using our definition of nifekalant as "effective", nifekalant was found to be effective in $25(83 \%)$ of the 30 patients in the nifekalant group. A termination effect was observed in one pulseless VT patient among 25 patients and a preventive effect was observed in the 24 remaining patients.

Effect of nifekalant on survival: In the nifekalant group, the mortality rate within 48 hours of occurrence was $7 \%(2 / 30)$, and $57 \%$ (17/30) of the patients in this group were discharged from the hospital.

Figure 3 illustrates the effect of nifekalant on survival. The rates of death within 48 hours of occurrence of VT/VF were significantly lower in the nifekalant group $(7 \%, 2 / 30)$ than in the control group $(27 \%, 9 / 33 ; P<0.03)$.

As an adverse effect of nifekalant, QT prolongation was observed in two patients. In one patient the QTc interval exceeded 0.65 seconds at a maintenance dose of $0.37 \mathrm{mg} / \mathrm{kg} \mathrm{BW} / \mathrm{h}$. Nifekalant administration was immediately discontinued and the QTc interval returned to the normal value. In another patient in which the QTc interval exceeded 0.62 seconds at a maintenance dose of $0.34 \mathrm{mg} / \mathrm{kg}$ 


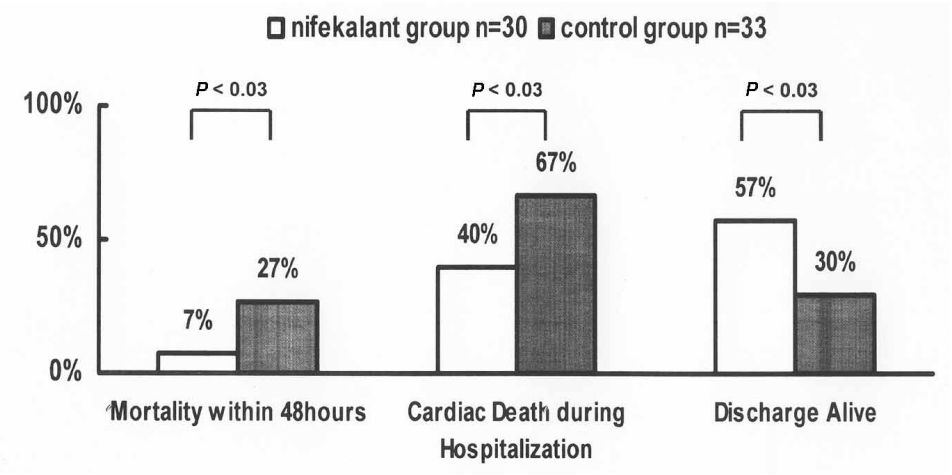

Figure 3. Comparison of nifekalant group and control group with respect to mortality rate within 48 hours, percentage of cardiac deaths during hospitalization, and percentage of discharges.

$\mathrm{BW} / \mathrm{h}$, torsade de pointes was transiently induced. DC countershock and the infusion of magnesium sulfate restored sinus rhythm. These two patients had impaired left ventricular function and mild renal insufficiency was observed in the case of torsade de pointes. Both were subsequently discharged from the hospital.

The total number of deaths was 36, including 34 cardiac deaths and 2 from subarachnoid hemorrhage and pneumonia. In the 34 cardiac deaths, 10 patients ( 2 in the nifekalant group, 8 in the control group) died due to uncontrollable VT/VF and 24 patients (10 in the nifekalant group, 14 in the control group) died mainly due to heart failure. The rates of cardiac death during hospitalization were also significantly lower in the nifekalant group $(40 \%, 12 / 30)$ than in the control group $(67 \%, 22 / 33 ; P<0.03)$. The rate of survival to hospital discharge was significantly higher in the nifekalant group $(57 \%, 17 / 30)$ than in the control group (30\%, 10/33; $P<0.03)$.

Univariate analysis revealed that nifekalant use and revascularization therapy were the factors that most significantly lowered the rate of mortality within 48 hours of occurrence of VT/VF (Table III). Multivariate analysis showed the factors that significantly affected mortality within 48 hours of onset of VT/VF were nifekalant use (odds ratio for mortality within 48 hours, 0.12; 95\% CI, 0.02 to $0.77 ; P=0.026$ ) and revascularization therapy (odds ratio for mortality within 48 hours, $0.12 ; 95 \% \mathrm{CI}, 0.02$ to $0.77 ; P=0.025$ ) (Table IV).

Concerning cardiac death during hospitalization, nifekalant use was the only factor that significantly improved the prognosis on univariate and multivariate analysis (odds ratio for cardiac death, $0.26 ; 95 \% \mathrm{CI}, 0.07$ to $0.95 ; P=0.041$ ). 
Table III. Univariate Analysis of Mortality Within 48 Hours

\begin{tabular}{lccc}
\hline & $\begin{array}{c}\text { Yes }(\%) \\
n=11\end{array}$ & $\begin{array}{c}\text { No }(\%) \\
n=52\end{array}$ & $P$ value \\
\hline Age $>75$ y.o & $5(45 \%)$ & $11(21 \%)$ & 0.09 \\
Cre $>2.0 \mathrm{mg} / \mathrm{dL}$ & $2(18 \%)$ & $15(29 \%)$ & 0.47 \\
HF & $11(100 \%)$ & $40(77 \%)$ & 0.08 \\
LVEF $\leq 35 \%$ & $5(45 \%)$ & $21(40 \%)$ & 0.76 \\
ACS & $7(64 \%)$ & $39(75 \%)$ & 0.44 \\
LMTD & $0(0 \%)$ & $10(19 \%)$ & 0.11 \\
MVD & $7(64 \%)$ & $25(48 \%)$ & 0.35 \\
Administration of nifekalant & $2(18 \%)$ & $28(54 \%)$ & 0.03 \\
Administration of lidocaine & $8(73 \%)$ & $48(92 \%)$ & 0.06 \\
Revascularization therapy & $7(64 \%)$ & $46(88 \%)$ & 0.04 \\
\hline
\end{tabular}

Abbreviations and meanings of terms in this table are the same as for Tables I and II.

Table IV. Multivariate Analysis of Mortality Within 48 Hours

$$
n=63
$$

\begin{tabular}{lccc}
\hline & $\begin{array}{c}\text { Odds ratio for } \\
\text { mortality within } 48 \mathrm{hrs}\end{array}$ & $(95 \% \mathrm{CI})$ & $P$ value \\
\hline Administration of nifekalant & 0.12 & $(0.02-0.77)$ & 0.026 \\
Revascularization therapy & 0.12 & $(0.02-0.77)$ & 0.025 \\
\hline
\end{tabular}

$\mathrm{CI}=$ confidence interval.

Other abbreviations and meanings of terms in this table are the same as for Table II.

While factors significantly increasing the rate of cardiac death on univariate analysis were heart failure, low LVEF, and LMTD, those on multivariate analysis were heart failure (odds ratio for cardiac death, 7.46; 95\% CI, 1.23 to $45.12 ; P=$ 0.029) and low LVEF (odds ratio for cardiac death, 6.54; 95\% CI, 1.64 to 26.03; $P=0.008$ ) (Table V, VI).

\section{DISCUSSION}

The efficacy of lidocaine in patients with cardiopulmonary arrest has been questioned, ${ }^{16)}$ since it is thought that lidocaine increases the risk of cessation of cardiac contraction in patients with VT/VF when cardiopulmonary arrest occurs. ${ }^{17)}$ On the other hand, the "Guidelines 2000 for Cardiopulumonary Resuscitation and Emergency Cardiovascular Care" recommend the use of amiodarone, a class III agent according to the Vaughan Williams classification, in patients in 
Table V. Univariate Analysis of Cardiac Death

\begin{tabular}{lccc}
\hline & $\begin{array}{c}\text { Yes }(\%) \\
n=34\end{array}$ & $\begin{array}{c}\text { No }(\%) \\
n=29\end{array}$ & $P$ value \\
\hline Age $>75 \mathrm{y} .0$ & $10(29 \%)$ & $6(21 \%)$ & 0.428 \\
Cre $>2.0 \mathrm{mg} / \mathrm{dL}$ & $10(29 \%)$ & $7(24 \%)$ & 0.638 \\
$\mathrm{HF}$ & $32(94 \%)$ & $19(66 \%)$ & 0.004 \\
LVEF $\leqq 35 \%$ & $20(59 \%)$ & $6(21 \%)$ & 0.002 \\
ACS & $27(79 \%)$ & $19(66 \%)$ & 0.216 \\
LMTD & $9(26 \%)$ & $1(3 \%)$ & 0.013 \\
MVD & $21(62 \%)$ & $11(38 \%)$ & 0.593 \\
Administration of nifekalant & $12(35 \%)$ & $18(62 \%)$ & 0.034 \\
Administration of lidocaine & $31(91 \%)$ & $25(86 \%)$ & 0.532 \\
Revascularization therapy & $29(85 \%)$ & $24(83 \%)$ & 0.784 \\
\hline
\end{tabular}

Abbreviations and meanings of terms in this table are the same as for Tables I and II.

Table VI. Multivariate Analysis of Cardiac Death

\begin{tabular}{lccc} 
& \multicolumn{3}{l}{$n=63$} \\
& $\begin{array}{l}\text { Odds Ratio for } \\
\text { Cardiac Death }\end{array}$ & $(95 \%$ CI $)$ & $P$ value \\
\hline HF & 7.46 & $(1.23-45.12)$ & 0.029 \\
LVEF $\leqq 35 \%$ & 6.54 & $(1.64-26.03)$ & 0.008 \\
LMTD & 6.00 & $(0.56-64.43)$ & 0.139 \\
Administration of nifekalant & 0.26 & $(0.07-0.95)$ & 0.041 \\
\hline
\end{tabular}

$\mathrm{CI}=$ confidence interval.

Other abbreviations and meanings of terms in this table are the same as for Table I.

the early phase after out-of-hospital refractory VF or pulseless VT. ${ }^{18)}$ Also, nifekalant, a class III antiarrhythmic agent, is thought to lower the defibrillation threshold, and strongly inhibits reentry tachyarrhythmia. As our findings show, nifekalant was effective for DC resistant VT/VF in IHD patients, and affected their prognosis favorably.

Takenaka, et $a l^{19)}$ reported that nifekalant is effective in treating malignant ventricular tachyarrhythmia following AMI with severe ventricular dysfunction that is refractory to class $\mathrm{Ia}$ and $\mathrm{Ib}$ antiarrhythmic agents. In another report, Igawa, et al showed that nifekalant could be an alternative drug for suppression of VT in patients with OMI. ${ }^{20)}$ It is possible the arrhythmogenic mechanisms may differ between ACS and OMI. However, we did not find any differences between ACS and OMI in the efficacy of nifekalant although the number of patients was limited. In our study, nifekalant was found to be effective for VT/VF in 25 (83\%) 
of 30 IHD patients resistant to DC countershock. Among these 30 patients, 26 (87\%) were administered lidocaine before nifekalant use. This efficacy rate is almost the same as that of the study reported by Amino on the efficacy of nifekalant for life-threatening ventricular tachyarrhythmia in patients resistant to lidocaine. ${ }^{21)}$ In four of these 25 patients, the recurrence of ventricular tachyarrhythmia could be prevented only by combining a loading dose of nifekalant and DC countershock, and did not require continuous administration of nifekalant. Furthermore, one of the remaining 21 patients recovered to sinus rhythm from pulseless VT with a loading dose of nifekalant alone without DC countershock. These results indicate that nifekalant can effectively lower the defibrillation threshold and terminate the ischemia-induced ventricular tachyarrhythmia at a relatively low dose.

This agent does, however, sometimes induce torsade de pointes ${ }^{19)}$ as a result of overdose. In our study, adverse events reported in association with nifekalant administration were marked QTc interval prolongation in two patients, one of whom experienced torsade de pointes. In patients with unstable hemodynamics, and renal and/or liver dysfunction, in particular, whose ability to metabolize drugs is often decreased, careful monitoring of the QTc interval is necessary.

Study limitations: It is important to recognize that this study was performed retrospectively at one facility with a small number of subjects. We failed to completely remove potential bias associated with differences in patient baseline characteristics and treatments because we did not use a randomized design and the observation period differed between the two groups. Although the antiarrhythmic agents administered other than nifekalant and the catheterization methods were not different between the two groups, improvement of our techniques for circulatory assistance may have contributed to the difference in the results.

Conclusion: Nifekalant is effective and relatively safe in treating DC countershock-resistant ventricular arrhythmia in patients with IHD, and improves their prognoses.

\section{REFERENCES}

1. Eisenberg MS, Horwood BT, Cummins RO, Reynolds-Haertle R, Hearne TR. Cardiac arrest and resuscitation: a tale of 29 cities. Ann Emerg Med 1990; 19: 179-86.

2. Eisenberg MS, Cummins RO, Damon S, Larsen MP, Hearne TR. Survival rates from out-of-hospital cardiac arrest: recommendations for uniform definitions and data to report. Ann Emerg Med 1990; 19: 1249-59.

3. Weaver WD, Copass MK, Bufi D, Ray R, Hallstrom AP, Cobb LA. Improved neurologic recovery and survival after early defibrillation. Circulation 1984; 69: 943-8.

4. Larsen MP, Eisenberg MS, Cummins RO, Hallstrom AP. Predicting survival from out-of-hospital cardiac arrest: a graphic model. Ann Emerg Med 1993; 22: 1652-8.

5. Stults KR, Brown DD, Schug VL, Bean JA. Prehospital defibrillation performed by emergency medical technicians in rural communities. N Engl J Med 1984; 310: 219-23. 
6. Nakaya H, Tohse N, Takeda Y, Kanno M. Effects of MS-551, a new class III antiarrhythmic drug, on action potential and membrane currents in rabbit ventricular myocytes. Br J Pharmacol 1993; 109: 157-63.

7. Kamiya J, Ishii M, Yoshihara K, Oyabe A, Banno H, Katakami T. MS-551: pharmacological profile of a novel class III antiarrhythmic agent. Drug Dev Res 1993; 30: 37-44.

8. Chen J, Komori S, Li B, Tamura K, Hashimoto K. IK independent class III actions of MS-551 compared with sematilide and dofetilide during reperfusion in anaesthetized rats. Br J Pharmacol 1996; 119: 937-42.

9. Martin DK, Nakaya Y, Wyse KR, Bursill JA, West PD, Campbell TJ. Inhibition of ATP-sensitive potassium channels in cardiac myocytes by the novel class III antiarrhythmic agent MS-551. Pharmacol Toxicol 1995; 77: 65-70.

10. Nakaya H, Uemura H. Electropharmacology of nifekalant, a new class III antiarrythmic drug. Cardiovasc Drug Rev 1998; 16: 133-44.

11. Kondoh K, Hashimoto H, Nishiyama H, et al. Effects of MS-511, a new class III antiarrhythmic drug, on programmed stimulation-induced ventricular arrhythmias, electrophysiology, and hemodynamics in a canine myocardial infarction model. J Cardiovasc Pharmacol 1994; 23: 674-80.

12. Tanabe $\mathrm{T}$, Iwamoto $\mathrm{T}$, Iwata $\mathrm{O}$, et al. Electrophysiologic and blood-flow responses in the endocardium and epicardium to disopyramide and MS-511 during myocardial ischemia in the dog. J Cardiovasc Pharmacol 1999; 34: 275-86

13. Yamada A, Motomura S, Hashimoto K. Comparison of direct negative chronotropic and positive inotropic effects of sematilide to those of E-4301 and MS-551 and the reverse frequency-dependent prolongation of cardiac refractoriness of sematilide. J Cardiovasc Pharmacol 1996; 27: 159-66.

14. Hayashi H, Fujiki A, Tani M, Usui M, Inoue H. Different effects of class Ic and III antiarrhythmic drugs on vagotonic atrial fibrillation in the canine heart. J Cardiovasc Pharmacol 1998; 31: 101-7.

15. Murakawa Y, Yamashita T, Kanese Y, Omata M. Can a class III antiarrhythmic drug improve electrical defibrillation efficacy during ventricular fibrillation? J Am Coll Cardiol 1997; 29: 688-92.

16. van Walraven C, Stiell IG, Wells GA, Hebert PC, Vandemheen K. Do advanced cardiac life support drugs increase resuscitation rates from in-hospital cardiac arrest? The OTAC Study Group. Ann Emerg Med 1998; 32: 544-53.

17. Dorian P, Cass D, Schwartz B, Cooper R, Gelaznikas R, Barr A. Amiodarone as compared with lidocaine for shock-resistant ventricular fibrillation. N Engl J Med 2002; 346: 884-90.

18. Guidelines 2000 for Cardiopulumonary Resuscitation and Emergency Cardiovascular Care: An International Consensus on Science. 6. Advanced cardiovascular life support. 5. Pharmacology I: agents for arrhythmias. Circulation 2000; 102: Suppl I:I-112-28.

19. Takenaka K, Yasuda S, Miyazaki S, et al. Initial experience with nifekalant hydrochloride (MS-551), a novel class III antiarrhythmic agent, in patients with acute extensive infarction and severe ventricular dysfunction. Jpn Circ J 2001; 65: 60-2.

20. Igawa M, Aonuma K, Okamoto Y, Hiroe M, Hiraoka M, Isobe M. Anti-arrhythmic efficacy of nifekalant hydrochloride, a pure class III anti-arrhythmic agent, in patients with healed myocardial infarction and inducible sustained ventricular tachycardia. J Cardiovasc Pharmacol 2002; 40: 735-42.

21. Amino M, Yoshioka K, Iwata O, et al. Efficacy of nifekalant hydrochloride for life-threatening ventricular tachyarrhythmias in patients with resistance to lidocaine: a study of patients with out-of-hospital cardiac arrest. J Cardiol 2003; 41: 127-34. 